\title{
Microcomb-based RF transversal filters
}

\author{
Xingyuan Xu, ${ }^{1}$ Mengxi Tan, ${ }^{1}$ Jiayang Wu, ${ }^{1}$ Thach G. Nguyen, ${ }^{2}$ Sai T. Chu, ${ }^{3}$ Brent E. Little, ${ }^{4}$ Roberto \\ Morandotti, ${ }^{5,6,7}$ Arnan Mitchell, ${ }^{2}$ and David J. Moss ${ }^{1, *}$ \\ ${ }^{1}$ Centre for Micro-Photonics, Swinbume University of Technology, Hawthorn, VIC 3122, Australia \\ ${ }^{2}$ ARC Centre of Excellence for Ultrahigh-bandwidth Devices for Optical Systems (CUDOS), RMIT University, Melbourne, VIC 3001, Australia \\ ${ }^{3}$ Department of Physics and Material Science, City University of Hong Kong, Tat Chee Avenue, Hong Kong, China. \\ ${ }^{4}$ State Key Laboratory of Transient Optics and Photonics, Xi'an Institute of Optics and Precision Mechanics, Chinese Academy of Science, Xi'an, \\ China. \\ ${ }^{5}$ INRS-Énergie, Matériaux et Télécommunications, 1650 Boulevard Lionel-Boulet, Varennes, Québec, J3X 1S2, Canada. \\ ${ }^{6}$ National Research University of Information Technologies, Mechanics and Optics, St. Petersburg, Russia. \\ ${ }^{7}$ Institute of Fundamental and Frontier Sciences, University of Electronic Science and Technology of China, Chengdu 610054, China. \\ *dmoss@swin.edu.au
}

\begin{abstract}
We demonstrate high-Q RF filters based on a $49 \mathrm{GHz}-\mathrm{FSR}$ integrated microcomb source that provides 80 wavelengths across the C-band. A $Q$ factor of 73.7 and an out-of-band rejection of $48.9 \mathrm{~dB}$ are demonstrated.
\end{abstract}

\section{Introduction}

RF filters are one of the basic and most commonly used components in modern electronic systems [1-3]. While electronic approaches are limited by the "electrical bottleneck" [4], photonic RF filters offer greater performance and many other advantages. Transversal photonic RF filters [5-10] generate weighted and progressively delayed replicas of an input RF signal in the optical domain and then combine them together upon photo-detection. They are capable of achieving arbitrary RF transfer functions by simply changing the tap weights, and are thus an attractive because of their extreme versatility. In this paper, we demonstrate a micro-comb [11] based photonic RF transversal filter with a record high number of taps consisting of 80 wavelengths over the C-band. This resulted in a $Q_{\mathrm{RF}}$ factor for the RF bandpass filter four times higher than previous results [12]. Further, by programming and shaping the Kerr optical micro-comb, we achieve RF filters with a high out-of-band rejection of up to $48.9 \mathrm{~dB}$ using Gaussian apodization. Our experimental results agree well with theory, verifying the feasibility of our approach towards the realization of high performance advanced adaptive RF transversal filters with potentially reduced cost, footprint, and complexity.

\section{Theory and Experimental results}

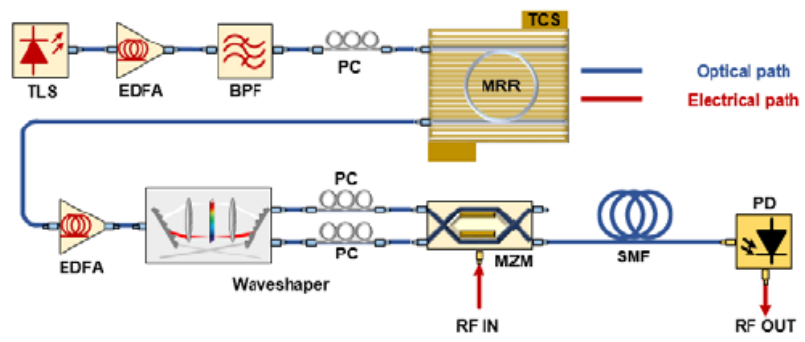

Fig. 1. Schematic diagram of the transversal RF filter based on microcombs. TLS: tunable laser source. EDFA: erbium-doped fibre amplifier. PC polarization controller. BPF: optical bandpass filter. TCS: temperature controller stage. MZM: Mach-Zehnder modulator. SMF: single mode fibre PD: photodetector.

Figure 1 shows a schematic diagram of the 80-tap transversal filter based on micro-combs. The soliton crystal comb was then spectrally shaped via a two-stage optical spectral shaper (Finisar, Waveshaper 4000S) in order to enable a larger dynamic range for the comb shape and higher shaping accuracy than a single stage [7]. The shaped comb lines were then fed into a dual input optical Mach-Zehnder modulator (MZM) biased at quadrature to yield both positive and negative taps, and then through $\sim 5-\mathrm{km}$ of standard single mode fibre (SMF) to provide the progressive tap delays. The dispersion of the SMF was $\sim 17.4 \mathrm{ps} /(\mathrm{nm} \cdot \mathrm{km})$, corresponding to a time delay $T$ of $\sim 34.8$ ps between adjacent taps, yielding an $F S R_{\mathrm{RF}}$ of $\sim 28.73 \mathrm{GHz}$ for the transversal filter.

To demonstrate the $Q_{\mathrm{RF}}$ factor enhancement brought about by the microcomb's large number of wavelengths, we implemented a low-pass sinc filter (i.e., $\left.h_{\text {sinc }}(n)=1\right)$ with different numbers of taps. The 3 -dB bandwidth $\left(B W_{\text {sinc }}\right)$ was measured to calculate the corresponding $Q_{\mathrm{RF}}$ factor $\left(Q_{\mathrm{RF}}=B W_{\mathrm{sinc}} / F S R_{\mathrm{RF}}\right)$. The $\mathrm{RF}$ transmission spectra of the sinc 
filter (Fig. 3(b)), measured by a vector network analyser (VNA, Anritsu 37369A), showed good agreement with theory (Fig. 3(a)). The measured $B W_{\text {sinc }}$ decreased from 3.962 to $0.236 \mathrm{GHz}$ when the tap number was increased from 2 to 80 , indicating a greatly enhanced $Q_{\mathrm{RF}}$ of up to 73.7 with 80 taps.

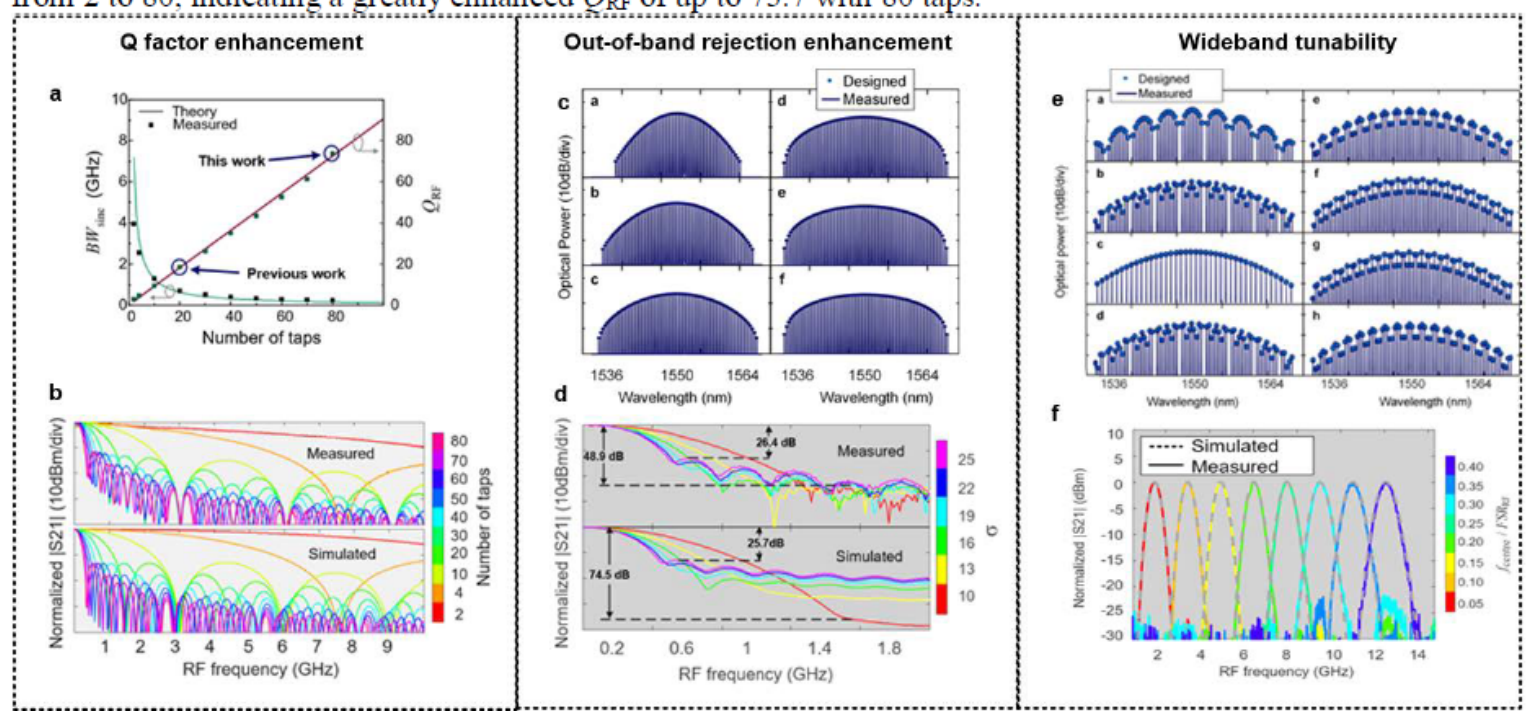

Fig. 2. (a) Calculated relationship between $\mathrm{Q}_{\mathrm{RF}}$ factor and the number of taps. (b) RF transmission spectra of the sinc filter. (c) Optical and (d) RF transmission spectra of the Gaussian-apodized sinc filter. (e) Optical and (f) RF transmission spectra of the centre-frequency-tunable filter

To improve the out-of-band rejection of the transversal filter, a Gaussian apodization was applied to the sinc filter, with a main-to-secondary sidelobe ratio of up to $48.9 \mathrm{~dB}$ (Fig. 3(c, d)). In order to demonstrate the tunability of the 80-tap transversal filter's centre frequency, the tap coefficients of the Gaussian-apodized sinc filter were multiplied by a sine function to shift the RF transmission spectrum. The corresponding RF transmission spectra (Fig. 3(f) ) shows a tunable centre frequency ranging from $0.05 \times F S R_{\mathrm{RF}}=1.4 \mathrm{GHz}$ to $0.40 \times F S R_{\mathrm{RF}}=11.5 \mathrm{GHz}$ with a relatively high MSSR of $>25 \mathrm{~dB}$, occupying $90 \%$ of the Nyquist band.

\section{Conclusion}

We demonstrate a microcomb-based photonic RF transversal filters with 80 taps over the C-band. We achieve $Q_{\mathrm{RF}}$ factors that are four times larger than previously demonstrated, as well as a high out-of-band rejection of up to 48.9 $\mathrm{dB}$ using Gaussian apodization. The experimental results agree well with theory, verifying that our transversal filter is a competitive approach towards achieving advanced adaptive RF transversal filters with broad operational bandwidths, high frequency selectivity, high reconfigurability, and potentially reduced cost and footprint, all of which are critical issues for modern communications systems.

\section{References}

[1] J. Capmany, B. Ortega, and D. Pastor, “A tutorial on microwave photonic filters," Journal of Lightwave Technol., vol. 24, no. 1, pp. 201-229, 2006.

[2] J. Capmany, and D. Novak, "Microwave photonics combines two worlds," Nat. Photonics, vol. 1, no. 6, pp. 319-330, 2007.

[3] J. P. Yao, "Microwave photonics," Journal of Lightwave Technol., vol. 27, no. 1-4, pp. 314-335, Jan-Feb. 2009.

[4] X. Xu et al., "Reconfigurable broadband microwave photonic intensity differentiator based on an integrated optical frequency comb source," APL Photonics, vol. 2, no. 9, 096104. 2017.

[5] X. Xu et al., "Photonic microwave true time delays for phased array antennas using a $49 \mathrm{GHz}$ FSR integrated optical micro-comb source," Photonics Res, vol. 6, no. 5, pp. B30-B36, May. 2018.

[6] X. Xu et al, "Photonic RF phase-encoded signal generation with a microcomb source", Journal of Lightwave Technology vol. 38, no.7, pp. 1722-1727. 2020. DOI:10.1109/JLT.2019.2958564.

[7] M. Tan et al., "Microwave and RF photonic fractional Hilbert transformer based on a 50GHz Kerr micro-comb," Journal of Lightwave Technology, vol. 37, no. 24, pp. 6097 - 6104. 2019. DOI: 10.1109/JLT.2019.2946606

[8] X. Xu et al., "Advanced Adaptive Photonic RF Filters with 80 Taps Based on an Integrated Optical Micro-Comb Source," Journal of Lightwave Technology, vol. 37, no. 4, pp. 1288-1295. 2019.

[9] X. Xu et al., "High performance RF filters via bandwidth scaling with Kerr micro-combs," APL Photonics, vol. 4, no. 2, pp. 026102.2019.

[10] X. Xu et al., "Broadband RF Channelizer based on an Integrated Optical Frequency Kerr Comb Source," Journal of Lightwave Technology, vol. 36, no. 19, pp.4519-4526. 2018.

[11] D. J. Moss, R. Morandotti, A. L. Gaeta, and M. Lipson, "New CMOS-compatible platforms based on silicon nitride and Hydex for nonlinear optics," Nat. Photonics 7, 597-607 (2013).

[12] X. Xu et al., "Advanced RF and microwave functions based on an integrated optical frequency comb source," Opt. Express, vol. 26, no. 3, pp. 2569-2583, Feb. 2018.

[13] X. Xu, M. Tan, J. Wu, R. Morandotti, A. Mitchell, and D. J. Moss, "Microcomb-based photonic RF signal processing," IEEE Photonics Technology Letters vol. 31, no. 23, pp. 1854-1857. 2019. DOI: 10.1109/LPT.2019.2940497 